\title{
Presentación de una Guía para la Observación y Clasificación de la Conducta Verbal de los Terapeutas
}

\section{Presentation of an Observation and Classification Guide of Therapists' Verbal Behavior}

\author{
Héctor Fernández-Álvarez \\ Claudia Castañeiras \\ Alejandro Curtarelli \\ Fernando García \\ Beatriz Gómez \\ Aldana Lichtenberger \\ Sergi Corbella
}

Fundación Aiglé. Buenos Aires, Argentina

(Rec: 14 de enero 2014/ Acept: 28 febrero 2015)

\begin{abstract}
Resumen
El artículo presenta una guía de observación y clasificación de la conducta verbal de los terapeutas, la Guía de Observación de Terapeutas Aiglé (GOTA-v). Se revisan los estudios sobre la variable del terapeuta, especialmente el Estilo Personal y sus procedimientos de evaluación. Este nuevo instrumento fue aplicado a la transcripción de 44 sesiones de 12 terapeutas de Argentina con diferente orientación teórico-técnica y distinto grado de experiencia (bajo, medio y alto). Se delimitaron los textos de las sesiones en unidades de análisis y se clasificaron esas unidades con categorías pertenecientes a tres clases de actos de habla: Preguntas, afirmaciones e indicaciones. Se calculó el índice de acuerdo inter jueces de Kappa para cada clase. Se discuten los alcances y limitaciones del instrumento y sus posibilidades para la investigación en psicoterapia. Palabras Clave: Terapeutas, Conducta Verbal, Guía de Observación
\end{abstract}

\begin{abstract}
The article presents an observation and classification guide of therapists' verbal behavior, the Aiglé Therapists Observation Guide (ATOG-v). Studies on the therapist variables together with the assessment procedures are revisited. This new instrument was applied to the transcription of 44 sessions of 12 therapists in Argentina with different theoretical-technical orientation and different levels of experience (low, moderate and high). The session texts were delimited into analysis units and these units were classified with categories pertaining three types of speech acts: questions, statements and indications. The Kappa inter-judges index was applied for each class. The scope and limitations of the instrument and its possibilities for psychotherapy research are presented.

Key Words: Therapists, Verbal Behavior, Observation Guide
\end{abstract}




\section{Introducción}

En los últimos 50 años, la investigación empírica ha puesto sistemáticamente en evidencia la importancia central del terapeuta en la determinación de los procesos y resultados de la psicoterapia (Garfield, 1997; Beutler, Malik, Alimohamed, Harwood, Talebi, et al., 2004). Los hallazgos acerca del impacto de las habilidades y características de los terapeutas sobre la eficacia de los tratamientos, aunque limitados y controvertidos (Lambert yBergin, 1983) han incrementado el interés de los investigadores (Garfield, 1997) y diversos estudios proporcionan datos que reflejan este hecho (Huppert, Bufka, Barlow, Gorman, Sheary Woods, 2001; Barber, Crits-Christoph y Luborsky, 1996; Crits-Christoph y Mintz, 1991; Machado, Beutler y Greenberg, 1999).

En la revisión realizada por Beutler, Machado y Allstetter Neufeldt (1994) se observó que las variables del terapeuta predominantemente investigadas hacían referencia a aspectos demográficos, factores socio-profesionales y cognitivoafectivos, patrones de personalidad, bienestar emocional, valores, actitudes y creencias, atributos que favorecen la influencia social o persuasión y estilo terapéutico. Sin embargo, no se contaba con estudios empíricos que se centraran específicamente en las características del estilo personal del terapeuta y su impacto en relación a los procesos de cambio y recuperación de los pacientes asistidos. Para estos autores, el estilo del terapeuta constituye un estado objetivo específico de la terapia y se trataría de un componente con marcada estabilidad temporal y que modula el modo específico de aplicar cualquier procedimiento o técnica psicoterapéutica. Una versión posterior de la revisión más importante, publicada en el año 2004, no incorporó aportes sustantivos al respecto (Beutler et al., 2004). Básicamente se sostiene que el grado de compatibilidad entre el estilo terapéutico y las características específicas de los pacientes influiría en los resultados de la psicoterapia (Beutler et al., 2004).

El capítulo dedicado a los terapeutas en el libro Bergin and Garfield's Handbook of Psychotherapy and Behavior Change (Lambert, 2013) propone cambios sustantivos en el tratamiento del tema. Sus autores Baldwin y Imel (2013), se centran en los efectos de los terapeutas sobre la alianza, adherencia y resultados de la psicoterapia y, en ese sentido, sugieren mejorar el conocimiento de las diferencias intraterapeutas.

En cuanto al concepto de estilo, Lazarus y Folkman (1984) sostienen que: "Las concepciones estilísticas de la conducta tienen una cierta proximidad a los rasgos, pero difieren de ellos puesto que no constituyen una estructura puntual estable y todavía menos son considerados como entidades intrapsíquicas por ninguno de los investigadores". Los estilos se tratarían de formas conductuales que se desarrollan a partir de los rasgos y que actúan como disposiciones con una naturaleza multidimensional más versátil.

En la Fundación Aiglé se desarrolló un programa de investigación sobre el Estilo Personal del Terapeuta -EPT(Fernández-Álvarez, y García, 1998; Castañeiras, García, Lo Bianco y Fernández-Álvarez, 2006; García, Castañeiras, Gómez y Fernández-Álvarez, 2009; Fernández-Álvarez, Gómez y García, 2013). El constructo EPT se define como "el conjunto de características que el terapeuta pone en juego en cada acto de psicoterapia, modelando los atributos fundamentales de dicho acto" Fernández-Álvarez y García (1998). Este estilo está constituido por las condiciones singulares que conducen a un terapeuta a operar de un modo particular en su desempeño profesional, influye en la marcha de cada proceso terapéutico e incide sobre los resultados de las intervenciones, aunque en diferente grado según el tipo de paciente, la patología y el contexto de aplicación específico. En estos términos, el EPT hace referencia a un constructo multidimensional, conformado por un conjunto de funciones que en la práctica se llevan a cabo de manera integrada, y expresan las disposiciones, rasgos y actitudes que todo terapeuta pone en evidencia en su ejercicio profesional. Dichas funciones terapéuticas no pretenden abarcar en su totalidad la naturaleza del estilo personal del terapeuta, ni explicar todas las acciones que éste lleva a cabo, pero sí se proponen como un criterio útil para delimitar los principales ejes sobre los que se desarrolla el acto psicoterapéutico.

El constructo EPT ha sido operacionalizado a través de un inventario autodescriptivo diseñado para su evaluación: el EPT-C.Una descripción detallada de sus características y propiedades psicométricas se ha presentado con anterioridad (Fernández-Álvarez, García, Lo Bianco y Corbella Santomá, 2003; Castañeiras, Ledesma, García, y Fernández-Álvarez, 2008). Se trata de un instrumento con índices de confiabilidad satisfactorios y validez teórica.

García y Fernández-Álvarez (2007) realizaron una revisión de las investigaciones empíricas en las que se aplicó el instrumento. Los trabajos relevados incluyen: el estudio del EPT como variable moderadora de los resultados de la psicoterapia; diferencias en el EPT que presentan terapeutas que asisten a distintas poblaciones clínicas (Vega, 2006), diferencias entre terapeutas con distinto grado de experiencia y orientaciones teórico-técnicas; y estudios técnicos sobre la estabilidad temporal del estilo terapéutico. Otros estudios constataron las relaciones entre algunas de sus dimensiones, las características de los pacientes y su impacto en la alianza terapéutica y los resultados de los tratamientos. Estos últimos 
hallazgos también fueron corroborados en otro trabajo y en una muestra diferente (Corbella Santomá, 2002; Malik, Fleming, Penela, Harwood et al., 2002). Todo esto alienta a seguir investigando el estilo personal del terapeuta como una variable central de la díada paciente-terapeuta y su relación con los resultados.

Por otra parte, si bien el EPT-C ha probado poseer validez teórica (Fernández-Álvarez et al., 2003; Castañeiras et al., 2008) y empírica (Corbella, 2002; Malik et al., 2002), el inventario está sujeto al sesgo de esta modalidad de evaluación. Anastasi (1958), en una caracterización ya clásica, planteó el debate sobre este tema. Las respuestas a los inventarios corresponden a la percepción que los sujetos tienen de la realidad. En este sentido, las correlaciones entre las respuestas a cada ítem del test y otros aspectos del comportamiento de los terapeutas deberán establecerse por medios empíricos. En un trabajo más reciente Sternberg y Grigorenko (2001) proponen la utilización de operaciones metodológicas convergentes para estudiar un mismo fenómeno. Para incrementar el conocimiento del estilo personal del terapeuta, es necesario estudiar lo que los terapeutas realmente hacen durante las sesiones más allá de sus respuestas a inventarios de lápiz y papel.

En su etapa actual el programa de investigación está dedicado a ampliar el mapa comprensivo del estilo personal más allá de las funciones evaluadas por el EPT-C. El objetivo es estudiar el estilo personal a través de la observación de jueces externos. A estos fines se desarrolló un instrumento para observar y clasificar la conducta verbal de los terapeutas durante la sesión: la Guía de Observación del Terapeuta Aiglé - verbal (GOTA-v).

En la literatura de investigación en psicoterapia existen antecedentes sobre el estudio de las acciones verbales de los terapeutas durante las sesiones y se ofrecen clasificaciones según diferentes criterios (Roussos, Etchebarne y Waizmann, 2006).

Una revisión detallada de este tema se puede consultar en Valdés, Tomicic, Pérez y Krause (2010) donde se citan 13 trabajos de los últimos 30 años. Algunos sistemas de clasificación incluyen a pacientes y terapeutas (Valdés et al., 2010).

Esta Guía fue diseñada específicamente para estudiar las conductas verbales de los terapeutas durante las sesiones, con independencia de los aspectos relacionales y los efectos de las intervenciones. El objetivo fue identificar y clasificar sus aspectos más estilísticos.

\section{Método}

\section{Participantes y Procedimiento}

Se audio grabaron un total de 44 sesiones de psicoterapia correspondientes a 12 terapeutas de Argentina con diferente orientación teórico-técnica (comportamentalcognitiva, psicodinámica e integrativa) y distinto grado de experiencia (bajo: menos de 5 años, intermedio: entre 6 y 15 años y alto: más de 16 años). En el estudio se incluyeron sesiones de tratamientos con pacientes diagnosticados con trastornos emocionales y/o trastornos de personalidad. Se excluyeron pacientes severos $\mathrm{y} / \mathrm{o}$ con historia de adicciones, con el fin de utilizar sesiones en las cuales la intervención psicológica fuera el tratamiento de elección o principal. En todos los casos se consideró como criterio de selección que las sesiones fueran parte del curso del tratamiento, no entrevistas iniciales o de cierre.

Se transcribieron en su totalidad las sesiones grabadas y se delimitaron tres momentos: los 10 minutos iniciales, los 10 minutos centrales y los 10 minutos finales de cada sesión, es decir un total de 30 minutos. Se siguió esta secuencia porque se consideró que el inicio de una sesión, el desarrollo de la misma y el cierre abarcan los momentos representativos de la actuación profesional.

Una vez realizadas las transcripciones, se identificaron unidades de análisis (UA) para su codificación. Con ese propósito se utilizaron y adaptaron las reglas propuestas por Hill y O'Brien (1999). Este primer nivel de clasificación define los requisitos de un texto para establecer una cláusula como independiente, dependiente o subordinada. También estas reglas prescriben las condiciones de exclusión para las expresiones que no son consideradas cláusulas. Bajo este procedimiento se identificaron 6979 UA en el total de sesiones. La tarea fue realizada por jueces previamente entrenados, todos ellos terapeutas, que actuaron de manera independiente. En los casos de no acuerdo con respecto a la delimitación de una UA, se incorporó un tercer evaluador para resolver las discrepancias. Se elaboraron criterios para la segmentación del texto en UA (ver Anexo 1).

A continuación se desarrollaron categorías para la clasificación de estas UA. Para su construcción se revisaron los antecedentes de Hill y O'Brien (1999) y de Stiles (1992). La guía se organizó en tres clases generales de actos de habla, siguiendo la clasificación de Searle (1969): Preguntas, Afirmaciones e Indicaciones. Se excluyeron 
las Exclamaciones/Interjecciones, debido su aparición excepcional. Cada clase se desagregó en categorías. Estas categorías se definieron en función del contenido y de la intencionalidad de las acciones verbales de los terapeutas. Para su delimitación se desarrollaron criterios de inclusión y exclusión en un Glosario (ver Anexo 2), que consigna el sistema clasificatorio de la guía. El Glosario incluye las denominaciones de las clases de actos de habla y los códigos que identifican cada una de las categorías y subcategorías y aporta ejemplos representativos.

Durante el proceso de elaboración de la guía se realizaron administraciones de prueba que dieron lugar a depuraciones sucesivas. Posteriormente se diseñó la versión final de la Guía: GOTA-v, que se presenta en este artículo.

La tarea de clasificación de las categorías también fue realizada por dos jueces independientes. Sobre un total de 9 sesiones, 3 sesiones de 3 terapeutas, se establecieron índices Kappa de Cohen para cada categoría de conductas, como una medida de concordancia inter jueces y para controlar la varianza de error atribuible al sesgo de los observadores, entendido este como la tendencia de un observador a clasificar consistentemente ciertas categorías por sobre otras. Esto permite establecer la confiabilidad inter jueces del instrumento para su uso en distintos contextos de investigación y aplicación.

Un sistema clasificatorio como el que se presenta pone en evidencia una situación dilemática con respecto a cómo han de resolverse los desacuerdos entre observadores. En los casos de falta de acuerdo se incorporó un tercer evaluador. Para ello, se siguió el principio de parsimonia, que guía la decisión de los jueces en función del menor grado posible de inferencia al clasificar una conducta.

A modo de ejemplo, cuando se presentaban para optar entre las categorías: Retomar algo de lo que dice, escribe o habla el paciente (AR) o Ir más allá de los que dice el paciente (AMA), el criterio prescribe optar por AR.

\section{Resultados}

La GOTA-v permitió clasificar un total de 1789 Preguntas, 4838 Afirmaciones y 142 Indicaciones. El índice Kappa alcanzó un valor de .84 para las preguntas con .03 de DE, .64 para las afirmaciones con .034 de DE y .93 para las indicaciones con $.027 \mathrm{de}$ DE con un intervalo de confianza de .95 .

En las Figuras 1 y 2 se expone la distribución total de la clasificación realizada por categorías para las clases de Preguntas y Afirmaciones. En una frecuencia residual se ubicaron las Indicaciones que alcanzaron un $2.03 \%$ de aparición en la clasificación y un 3.01\% de UA de Actos sin clasificar (ASC).

La Figura 1 muestra la frecuencia de aparición de las distintas categorías de preguntas.

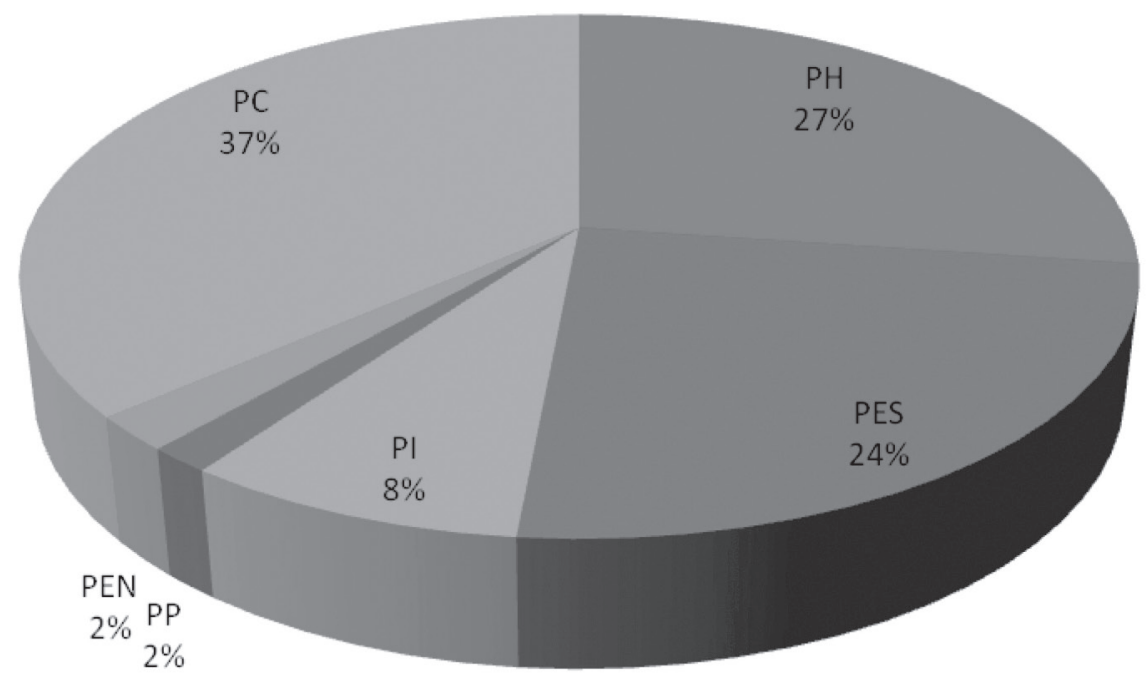

Figura 1. Distribución porcentual para Preguntas 


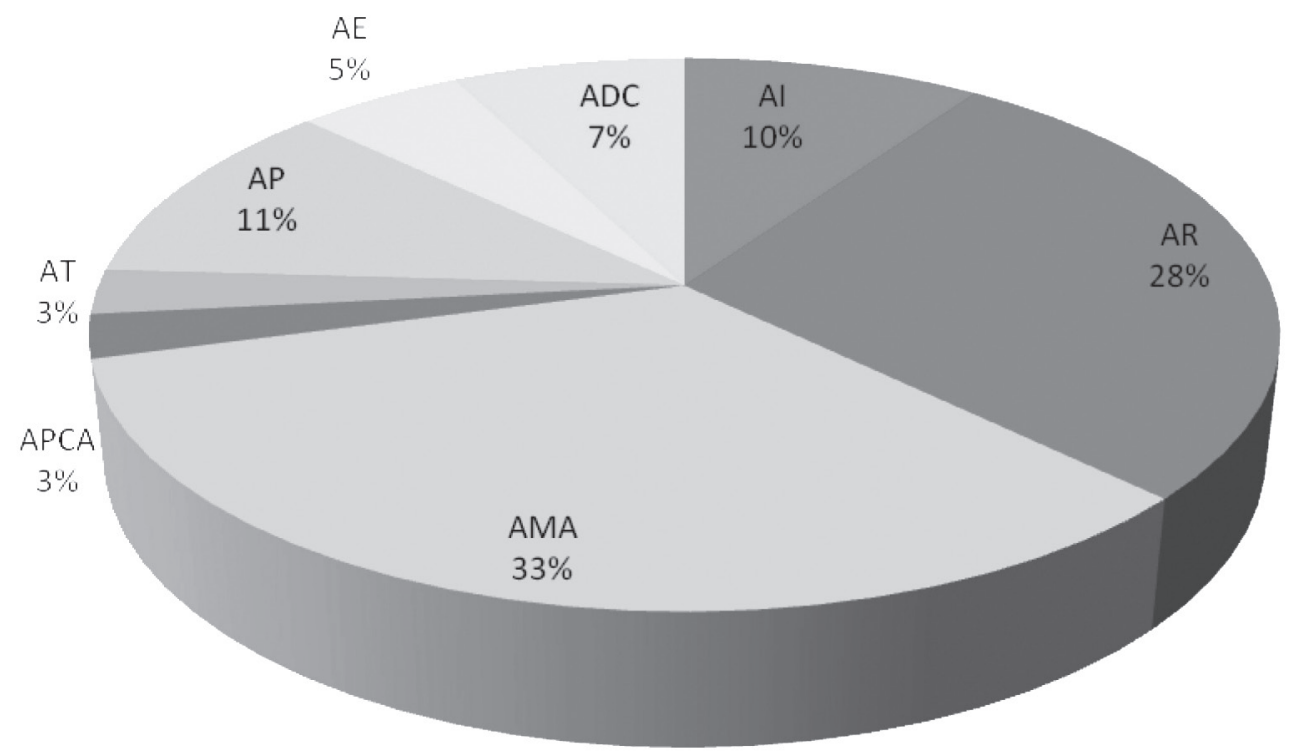

Figura 2. Distribución porcentual para Afirmaciones

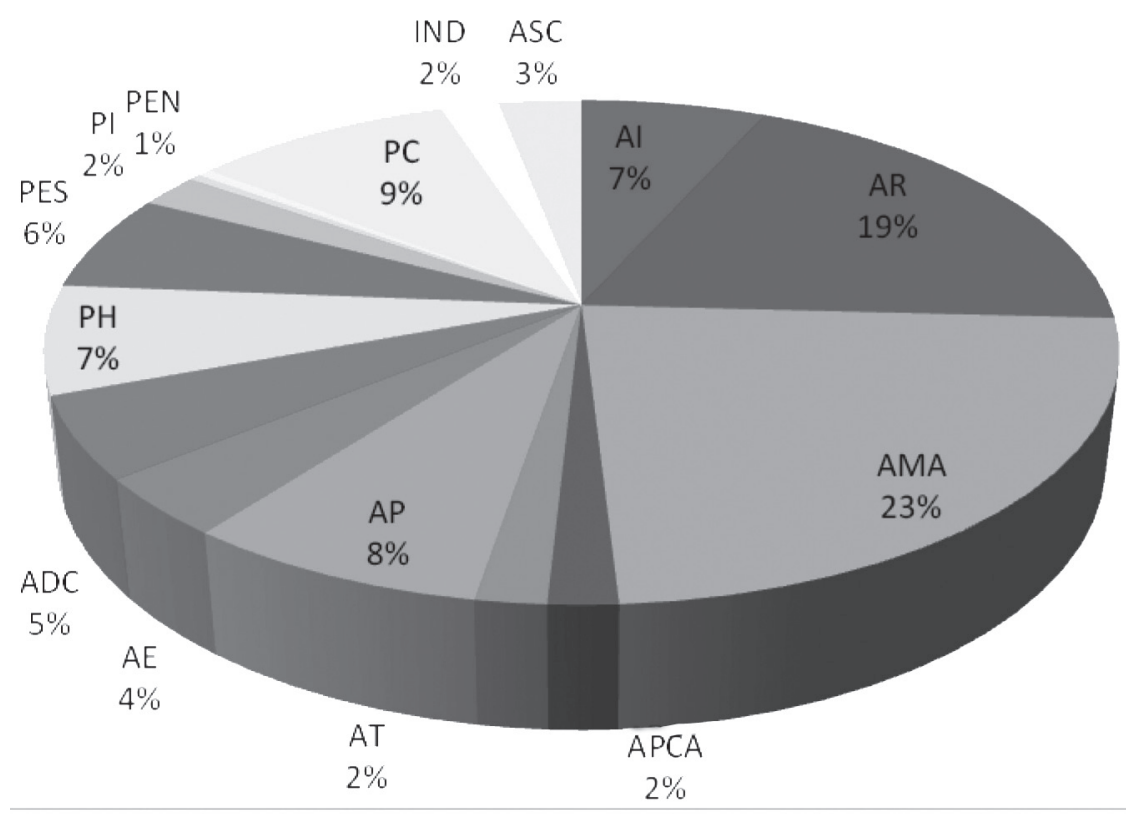

Figura 3. Distribución porcentual General

Como puede observarse, las preguntas de confirmación de recepción del mensaje (PC) fueron las de mayor frecuencia de aparición en los terapeutas, seguidas por preguntas sobre comportamientos, datos y hechos $(\mathrm{PH})$ y sobre la experiencia subjetiva (PES), que se define como los pensamientos, emociones, evaluaciones e intenciones del paciente de forma explícita respecto de un tema que está siendo tratado en la sesión, o de la relación terapéutica. En cuanto a las preguntas para interpretar y relacionar (PI), cuya función es reflexionar sobre cursos de pensamiento o de acción imaginarios, generar nuevas posibilidades o nuevas relaciones entre temas o aspectos de un tema, tuvieron una presencia sustancialmente menor y las referidas al proceso terapéutico (PP) y al encuadre (PEN) fueron residuales en las sesiones analizadas.

En el caso de las Afirmaciones (Figura 2), el 33\% de las clasificaciones correspondieron a afirmaciones en las que el 
terapeuta aporta un significado diferente al propuesto por el paciente (AMA) como es el caso de lo que se conoce técnicamente como reformulación o confrontación, o distintos grados de interpretación. El 28\% se refirió a AR, que son aquellas afirmaciones como repetir, parafrasear, recapitular o señalar algo de lo que dice, escribe o hace el paciente.

Con mucha menor frecuencia se clasificaron afirmaciones que buscan orientar y estructurar los distintos aspectos del proceso terapéutico (AP), que se basan en aportarle información general o específica al paciente sobre cuestiones clínicas (AI) y que buscan validar o valorar positivamente los pensamientos o emociones del paciente (ADC).

Con una frecuencia residual los terapeutas usan afirmaciones sobre el encuadre del tratamiento (AE), auto revelaciones sobre aspectos de la vida personal del terapeuta (AT), y recomendaciones y consejos que sugieren cursos de acción alternativo (APCA).

A continuación se presentan la distribución general (en porcentajes) de las categorías que incluye la guía.

En esta primera etapa se presenta la clasificación de intervenciones en las categorías más generales. En próxima etapa, se codificarán de manera diferenciada las subcategorías que han sido incluidas en el Glosario.

\section{Discusión}

El proceso de construcción y puesta a prueba de la Guía derivó en la versión que se presenta en este artículo.

La GOTA-v resulta un instrumento confiable para clasificar con un adecuado grado de precisión las conductas verbales de los terapeutas, en las modalidades Preguntas, Afirmaciones e Indicaciones. Tal como se mencionó en la introducción de este trabajo, el estudio de las variables del terapeuta ha presentado cambios en su desarrollo.

La naturaleza multidimensional atribuida al constructo del estilo personal del terapeuta y su relativa estabilidad temporal han dado lugar al estudio de distintos aspectos de su composición. En este sentido, contar con un instrumento estructurado que permita clasificar las conductas verbales de los terapeutas en sesión, constituye un avance en la evaluación de las características estilísticas en diferentes contextos de investigación y aplicación. La GOTA-v fue diseñada con esa intención.

Una limitación de este estudio radica en que la clasificación propuesta no pretende ser exhaustiva, dada la complejidad del fenómeno discursivo. Por otro lado, comunicación durante el proceso psicoterapéutico requiere considerar tanto los aspectos verbales como los no verbales. El hecho que esta Guía se centre en aspectos verbales exclusivamente no tiene una finalidad restrictiva y acotada del acto clínico, sino que busca ofrecer un recurso técnico de más clara delimitación que supone la interpretación de los aspectos no verbales en los terapeutas.

Asimismo, es importante incrementar la cantidad de casos clínicos por terapeuta y considerar el efecto modulador de otras variables sobre las intervenciones verbales, como por ejemplo la orientación teórico-técnica de los terapeutas, las condiciones clínicas y otras variables del paciente y el contexto. Será también de interés aplicar la GOTA-v a fragmentos de diálogos no clínicos con el objetivo de evaluar el grado de especificidad del uso de los modos verbales en contextos terapéuticos.

\section{Referencias}

Anastasi, A. (1958). Psychological Testing.New York: The Macmillan.

Baldwin, S. A., y Imel, Z. E. (2013). Therapist effects: Findings and methods. In M.J. Lambert (Ed.).Bergin and Garfield's Handbook of Psychotherapy and Behavior Change (pp. 258-297). Hoboken, N.J.: Wiley.

Barber, J. P., Crits-Christoph, P., y Luborsky, L. (1996). Effects of therapists adherence on patient outcome in brief dynamic therapy. Journal of Consulting and Clinical Psychology, 64, 619-622.

Beutler, L. E., Machado, P.P., y AllstetterNeufeldt, S. (1994) Therapist variables. In A. Bergin y S. Garfield (Eds.).Handbook of psychotherapy and behavior change.(pp.229-269). New York: Wiley.

Beutler, L., Malik, M., Alimohamed, S., Harwood, T. M., Talebi, H. et al., (2004).Therapist Variables.In M. J. Lambert (Ed.).Bergin and Garfield's Handbook of Psychotherapy and Behavior Change.(2nd Ed., pp. 227306). New York: Wiley.

Castañeiras, C., García, F., Lo Bianco, J., y Fernández-Álvarez, H. (2006). Modulating Effect of Experience and Theoretical-Technical Orientation on the Personal Style of the Therapist.PsychotherapyResearch, 16, 587-593.

Castañeiras, C., Ledesma, R., Garcia, F., y Fernández-Álvarez, H. (2008) Evaluación del Estilo Personal del Terapeuta: presentación de una versión abreviada del Cuestionario EPT-C. Terapia Psicológica, 26, $5-13$.

Corbella Santomá, S. (2002) Compatibilidad entre el estilo personal del terapeuta y el perfil personal del paciente. (Tesis doctoral). Facultat de Psicologia, Ciències de I'Educació i de I'EsportBlanquerna, UniversitatRamonLlull, Barcelona.

Crits-Christoph, P., y Mintz, J. (1991).Implications of therapist effects for the designs and analysis of comparative studies of psychotherapies. Journal of Consulting and Clinical Psychology, 59, 20-26.

Fernández-Álvarez, H., y García, F. (1998).El Estilo Personal del Terapeuta: Inventario para su evaluación. En S. Gril, A. Ibáñez, I. Mosca y P.L.R. Sousa (Eds.). Investigación en psicoterapia.(pp. 76-84). Pelotas, Brasil: Educat.

Fernández-Álvarez, H., García, F., Lo Bianco, J., y Corbella Santomá, S. (2003). Assessment Questionnaire on the Personal Style of Therapist. PST-Q.Clinical Psychology y Psychotherapy, 10, 116-125.

Fernández-Álvarez, H., Gómez, B., y García, F. (2013). Bridging the gap between research and practice in a clinical and training network: Aigle's Program.Psychotherapy Research, DOI: 10.1080/10503307.2013.856047.

García, F., Castañeiras, C., Gómez, B., y Fernández-Álvarez, H. (2009). New Developments on the Personal Style of the Therapist.40th SPR International Meeting.Santiago, Chile. 
García, F., y Fernández-Álvarez, H. (2007).Investigación empírica sobre el Estilo Personal del Terapeuta: Una actualización. Revista Argentina de ClínicaPsicológica, 16, 121-128.

Garfield, S. L. (1997). The therapist as neglected variable in Psychotherapy Research.Clinical Psychology: Science and practice, 4, 40-43.

Hill, C. y O’Brien, K.M. (1999). Helping Skills.Facilitating Exploration, Insight and Action. Washington D.C.: American Psychological Association.

Huppert, J. D., Bufka, L.F., Barlow, D.H., Gorman, J.M., Shear, M.K., y Woods, S.W. (2001). Therapists, therapists variables and cognitive behavioral therapy outcome in a multicenter trial for panic disorder. Journal of Consulting and Clinical Psychology, 69, 747-755.

Lambert, M. J. (2013).Bergin and Garfield's Handbook of Psychotherapy and Behavior Change.Hoboken, N.J.: Wiley.

Lambert, M. J., y Bergin, A.E. (1983).Therapist characteristics and their contribution to psychotherapy outcome.In C.E. Walker (Eds.).The handbook of Clinical Psychology.(Vol 1, pp. 205-241). Homewood, IL: Down Jones Irwin.

Lazarus, R., y Folkman, S. (1984).Stress, appraisal and coping. New york: Springer Publishing Company.

Machado, P.P., Beutler, L.E., y Greenberg, L.S. (1999). Emotion recognition in Psychotherapy: impact of therapist level of experience and emotional awareness, Journal of Clinical Psychology, 55, 39-57.

Malik, M.L., Fleming, J., Penela, V., Mark T. Harwood, M.T., Best, S., y Beutler, L. (2002). Which therapist for which client? The interaction of therapist variation and client characteristics in affecting rates of change: An effectiveness study, Paper presented at the 33rd Annual Meeting of the Society for Psychotherapy Research, San Francisco, USA.

Roussos, A., Etchebarne, I., y Waizmann, V. (2006). Un esquema clasificatorio para las intervenciones en psicoterapia. Anuario de la Universidad de Buenos Aires, XIII, 51-61.

Searle, J. (1969). Speech acts: an Essay in philosophy of language. Cambridge: University Press.

Sternberg, R. J., y Grigorenko, E. L. (2001). Unified Psychology.American Psychologist, 56,1069-1079.

Stiles, W. B. (1992).Describing talk: A taxonomy of verbal responses. Thousand Oaks: Sage.

Valdés, N., Tomicic, A., Pérez, J. C., y Krause, M. (2010).Sistema de Codificación de la Actividad Terapéutica (scat-1.0): Dimensiones y categorías de las acciones comunicacionales de pacientes y psicoterapeutas. Revista Argentina de Clínica Psicológica, 19, 117-130.

Vega, E. (2006). El psicoterapeuta en neonatología. Buenos Aires: Lugar Editorial. 


\section{Anexo 1- Criterios acerca de la puntuación de UA}

\section{Autonomía de la cláusula}

Este es el criterio fundamental para la delimitación de UA, y todo criterio debe subordinarse a este en caso que hubiere que tomarse una decisión. El poseer autonomía implica que cumple los requisitos para ser un acto de habla.

\section{Metodología para la delimitación de UA}

La cláusula, al abstraerse debe tener sentido por sí misma. $\mathrm{Ej}$.:

$\{$ Vos pudiste aprender $\}$ y \{pudiste discernir $\}$ Se separan

$\{$ Tenés otras herramientas y otros recursos para poder hacer algo distinto de lo que hacías.\} No se separa.

La cláusula no debe poder ser reducida a un pronombre o un adverbio ya que esto implicaría que forma parte de una sub-estructura de otra proposición
Ej.:

\{pudiste discernir la diferencia entre decoración y diseño $\}=\{$ pudiste discernir eso $\}$ O sea que "la diferencia entre decoración y diseño" no constituye una cláusula autónoma.

$\{$ Tenés otras herramientas y otros recursos para hacer algo distinto de lo que hacías $\}=\{$ Tenés otras herramientas $y$ otros recursos para eso $\}=\{$ Lastenés para eso $\}$

\section{Criterios de una cláusula autónoma:}

Verboides:

Si bien tienen significado, no suelen cumplir los requisitos para una UA.

Infinitivo.

$\{$ Hablar de todo aquello que veníamos hablando $\}$ No cumple los requisitos para ser UA. Es una estructura que tiene función de sustantivo.

$\{$ Hablarde todo aquello que veníamos hablando es lo que nos trajo hasta acá\} A diferencia de la anterior el poseer verbo permite que cumpla los criterios para ser un acto de habla.\}

Discriminación de UA en función de sus nexos (coordinantes o subordinantes) o los pronombres que las encabezan

\begin{tabular}{ll}
\hline Conjunciones Coordinativas: & Conjunciones Subordinadas \\
\hline SI / NO & DESPUÉS / ANTES / CUANDO / MIENTRAS / DONDE / \\
SINO & AUNQUE / \\
Ó/ O SEA QUE/ COMO QUE & COMO / TANTO COMO / \\
PERO & PORQUE* / POR CUANTO \\
Adverbios Conjuntivos: & "SI x... ENTONCES y..." \\
TAMBIÉN / ENTONCES / POR LO TANTO & ASI QUE / AL MENOS \\
AÚN / TODAVÍA & Conjunciones Correlativas \\
SIN EMBARGO / POR OTRO LADO / MÁS ALLÁ DE / & Tanto - tanto. Ni - ni. Ambos - y. No solo - pero. Si - o. \\
AUNQUE (si está en lugar de sin embargo) & O bien - O bien. \\
por consiguiente / consecuentemente / & Pronombres que suelen encabezarlas: \\
& QUIEN / CUAL / QUE \\
\hline
\end{tabular}

1. Delimitación de UA en función a decisiones tomadas previamente

\begin{tabular}{lll}
\hline & No Puntuar & Puntuar \\
\hline Mhm... aha... & Si acompasan el discurso del paciente & Si son respuesta a una pregunta \\
Claro; Ok; & Si acompasan el discurso del paciente & Si son respuesta a una pregunta \\
¿Eh?; ¿Mmmh?: & Si cumplen una función de apoyatura al hablar. & Si funcionan como pregunta acerca de lo que dijo el P \\
& & \\
No sé; qué sé yo. & Si cumplen una función de apoyatura al hablar. & Si es una afirmación del desconocimiento de algo por parte de T \\
Si. No. & Si acompasan o son un reflejo del discurso del P & Si su función es afirmar algo \\
Bien. & Si acompasan o son un reflejo del discurso del P & Si su función es calificar positivamente la conducta del P \\
\hline
\end{tabular}

Cláusulas Interpuestas. A menudo dentro de una cláusula aparece otra cláusula incrustada. En este caso se puntúan ambas como cláusulas válidas. 
Ej.:

\{Vos me contabas que estabas yendo / hace quince días me dijiste esto/ eh... más seguido a verlos\}

$\{$ La verdad es que por todo lo que vos me estás contando /¿No es cierto?/este tema estaría solucionado\}

\section{Anexo 2 - Glosario GOTA-v}

Esta guía fue diseñada para clasificar tres clases de actos de habla: Preguntas, Indicaciones y Afirmaciones. Para cada una de ellas se determinaron categorías con el fin de diferenciar las acciones realizadas por los terapeutas.

Los criterios de inclusión en cada clase fueron:

a) el modo verbal - para distinguir declaraciones imperativas de afirmaciones

b) la entonación ${ }^{1}$ - para distinguir preguntas de afirmaciones (Seco, 1999)

A continuación se exponen los criterios de inclusión para determinar la pertenencia a cada categoría y sub categoría.

\section{Preguntas:}

a) Cláusulas que cumplan las características de entonación propias del idioma Español.

b) Enunciados del tipo: quiero/quisiera/querría preguntarte $x$.

Se excluyen enunciados en los que una pregunta sea parte de un objeto directo o un predicativo.

Ejemplo: T: Es como si yo dijera ¿no será que soy yo? Decías ¿Qué hice yo para merecer esto? (Paráfrasis).

Las preguntas pueden ser Abiertas o Cerradas (excepto la categoría PC).

1) (PH) Preguntas sobre Hechos, Comportamientos y Datos: Con el fin de obtener información sobre acciones, situaciones y acontecimientos.

Ejemplos:

Abierta: ¿Qué haces a la mañana cuando te despiertas?

Cerrada: ¿La volviste a llamar?

2) (PES) Preguntas sobre la Experiencia Subjetiva:

Indagan sobre pensamientos, emociones, evaluaciones e intenciones del paciente de forma explícita respecto de un tema que está siendo tratado en la sesión o de la relación terapéutica.

Utilizan verbos mentalistas o refieren a conductas privadas (amar, ansiar, anticipar, confiar/desconfiar, evaluar,

Seco Reymundo, M. (1999) "Gramática Esencial de la Lengua Española de Manuel Seco”. Ed. Espasa Calpe S.A. (pp. 116-118). funcionar, creer, desear, dudar, esperar - pensar, sentir, sufrir, soñar, querer, odiar, proyectar, planificar, razonar, imaginar, etc.).

Se incluyen preguntas que utilizan los verbos ser, estar, resultar, etc.para indagar sobre estados mentales.

Ejemplo: ¿Cómo estás con el tema este?

Las preguntas sobre el encuadre o el proceso se puntúan PEN o PP respectivamente.

Esta categoría posee tres subcategorías:

2.1. Preguntas sobre pensamientos, emociones y evaluaciones del paciente sobre temas generales.

Ejemplo: ¿Qué estás pensando?

2.2. Preguntas sobre pensamientos, emociones y evaluaciones del paciente sobre la relación terapéutica. Ejemplo: ¿Te enojaste por lo que te dije?

2.3. Preguntas sobre las razones que da el paciente a distintos sucesos. Son formulaciones que si bien pueden no incluir un verbo mentalista, lo implican. Ejemplo: ¿Por qué te parece que él está reaccionando así ahora?, ¿Por qué él está reaccionando asi ahora?

Pueden ser abiertas o cerradas. Ejemplos:

Abierta: ¿Qué sentiste cuando te llamó?

Cerrada: ¿Te alivió poder hablarlo?

3) (PI) Preguntas para Interpretar o Relacionar: Su función es reflexionar sobre cursos de pensamiento o de acción imaginarios, generar nuevas posibilidades o nuevas relaciones entre temas o aspectos de un tema.

Ante la duda entre PI y PH o PES se puntúa PH o PES según corresponda.

Incluye cinco subcategorías:

3.1.Preguntas que sugieren Hipótesis Explicativas.

Ejemplo: ¿No será que en realidad la que no lo quiere llamar eres tú?

3.2. Preguntas que sugieren relaciones explícitas entre temas.

Ejemplo: ¿No se parece esto a lo que me contabas de la relación con tu papá?

3.3. Preguntas que sugieren la posibilidad de otras alternativas.

Ejemplo: ¿Por qué... necesariamente tenías que sentirte exigida y obligada? ¿No podría ser que él quisiera otra cosa?

3.4.Preguntas para confrontar. Suelen señalar en forma de pregunta los límites de una afirmación para dar cuenta de un sesgo del paciente.

Ejemplo: ¿No hay NINGUNA chance? 
3.5.Preguntas para sugerir un curso de acción alternativo.

Sugieren un curso de acción o una posibilidad no tenida en cuenta. Pueden tener una función retórica. Ejemplo: ¿No te parece que sería mejor enfrentarlo? Pueden ser abiertas o cerradas. Ejemplos:

Abierta: ¿Qué tendría que haber pasado para que las consecuencias fueran distintas?

Cerrada: ¿Preferís estar enojada antes que triste?

4) (PP) Preguntas sobre el Proceso Terapéutico: Su foco está puesto en las emociones, sentimientos, creencias, deseos, pensamientos o acciones del paciente sobre objetivos, medios, fases y evolución del tratamiento. (Aclaración: se considera como proceso terapéutico al proceso actual, no tratamientos anteriores. En caso de superposición entre PP, PH y PES, se puntúa PH o PES según corresponda).

Pueden ser abiertas o cerradas. Ejemplos:

Abierta: ¿Por qué te cuesta contarme lo que pasó?

Cerrada: ¿Te cuesta recordar lo que hablamos la semana pasada?

5) (PEN) Preguntas sobre el Encuadre: Referidas al dispositivo terapéutico en lo que respecta al acuerdo de fechas, horarios y honorarios.

Ejemplos:

Abierta: ¿Por qué llegaste tarde?

Cerrada: ¿Nos vemos en dos semanas?

6) (PC) Pregunta para confirmar recepción del mensaje: Suelen ser breves y se realizan en la mitad o al final de una intervención para constatar la recepción, comprensión y/o el acuerdo respecto a una afirmación del terapeuta. Ejemplo: Fue difícil escucharlo, ¿no? / ¿Se entiende?

Indicaciones:Aquellas instrucciones enunciadas por el terapeuta con respecto a la realización de un curso de acción. Se formulan en modo imperativo.

Ejemplo: Escúchame / Anota todo lo que creas que podrias hacer para sentirte mejor.

Afirmaciones: Aseveraciones.

1) (AI) Dar información:

Incluye dos subcategorías:

1.1. Del marco teórico/patología: Afirmaciones en las que se describe en términos genéricos y técnicos la naturaleza de procesos psicológicos, datos estadísticos, y todo aquello que implica el conocimiento teórico-técnico del terapeuta. Ejemplo: No todas las personas sienten lo mismo frente a una pérdida.
1.2.Información general:Enunciados en los que el terapeuta aporta datos sobre aspectos de la realidad cotidiana.

Ejemplo: Esa línea de trenes no está funcionando.

2. (AR) Repetir, Parafrasear, Recapitular o Señalar algo de lo que dice, escribe o hace el paciente:

Incluye cinco subcategorías:

2.1.Reflejo: Se repite algo que acaba de decir el paciente con las mismas palabras o de forma muy similar.

Ejemplo: P: No sé si todo, pero bastante bien. T: Ah, bastante bien.

2.2. Completar la Frase: El terapeuta anticipa lo que iba a decir el paciente o completa una frase en el mismo sentido dado por el paciente. En caso de duda con AMA se puntúa AR.

Ejemplo: P: Yo quería... T: Descansar. P: Sí.

2.3.Recapitular: El terapeuta hace una síntesis o resumen de lo que dijo el paciente en esa u otra sesión, o sobre eventos ocurridos anteriormente en el proceso terapéutico. Trae a la situación actual algo hablado anteriormente.

(Aclaración: se puntúa cuando el terapeuta alude explícitamente a algo que se habló anteriormente utilizando palabras como: Tu me decías; decíamos; Volvamos a...; Hablamos hace un rato....

Afirmaciones sobre fases, etapas o momentos del proceso terapéutico no pertenecen a esta categoría y se puntúan AP).

Ejemplo: Decias que fue doloroso sentirlo.

2.4. Paráfrasis: El terapeuta repite algo de lo dicho por el paciente empleando diferentes palabras sin modificar el significado. Refiere a aquello de lo que el paciente está hablando en ese momento. (Aclaración: puede tener una forma más abreviada y concisa que la expresada por el paciente. El terapeuta modifica por sinonimia las premisas o las conclusiones del paciente).

Ejemplo:P: Esta semana salimos el lunes, el jueves, el miércoles. T: Ahh, salieron tres días...

2.5. Señalamiento: Afirmaciones que destacan un elemento sobre otros dirigiendo la atención hacia ese punto. Pueden ser elementos no mencionados por el paciente, pero implicados en la lógica de lo que está diciendo sin cambiar las premisas o las conclusiones del razonamiento. 
(Aclaración: se pone el foco sobre reiteraciones, relaciones entre datos o las consecuencias lógicas de un razonamiento, pudiendo también comparar uno o más elementos del discurso del paciente con otro/s, destacando su similitud o su diferencia. Son declaraciones que podrían comenzar diciendo: Parece que... entonces esto implica que...). Ejemplo: Parece que siempre volvemos al mismo punto.

En caso de superposición con AE o AP se clasifica AE o AP.

3. (AMA) Ir más allá de lo que dice el paciente: El terapeuta aporta un significado diferente al propuesto por el paciente.

Incluye siete subcategorías:

3.1. Reformulación: El terapeuta ofrece una explicación distinta a la que maneja el paciente, cambiando la estructura de la frase y ofreciendo una definición alternativa, agrega elementos o cualidades no expresadas por el paciente. La explicación puede ser formulada en términos del lenguaje coloquial o con terminología teórico-técnica en la que se realiza una articulación entre el relato del paciente y el marco teórico del terapeuta.

Son oraciones que podrían empezar diciendo $E n$ realidad..., lo que pasa es que..., o sea...

(Aclaración: se diferencia de Dar Información en que se ofrece una hipótesis explicativa de lo que relata el paciente y no se trata de la rotulación de un elemento o la descripción de las características de un cierto proceso normal o patológico).

Ejemplo: En realidad, lo que no te permite salir de ese pensamiento es el enojo...

3.2. Metáfora: Afirmaciones en las que se emplean términos en sentido figurado o imágenes alegóricas con el fin de representar una idea propia o del paciente.

Ejemplo: Lo importante no es que llueva sino como protegerse.

3.3. Confrontación: Se afirma de manera explícita algo opuesto a lo que dice el paciente. Se incluyen también frases que incitan al paciente a poner en duda lo que dice.

Ejemplo: P: No puedo hacer nada con esto! T: No es que no puedes nada... Algo puedes...
3.4. Hipótesis Predictiva: El terapeuta afirma, con mayor o menor grado de certidumbre que algo sucederá de cierta forma.

Ejemplo: Me arriesgaría a decir que independientemente de lo que pase hoy te vas a sentir más aliviada.

3.5. Reflexiones sobre alternativas no tenidas en cuenta por el paciente. Suelen formularse empleando conjugaciones del verbo poder en modo condicional o en presente de indicativo y mencionando una o varias situaciones hipotéticas. (Aclaración: Se diferencia de Señalamiento porque aporta algo distinto de lo que dice el paciente. Se diferencia de Indicación porque no está formulada en modo imperativo ni tiene carácter instruccional. Si se superpone AMA y AP se puntúa AP).

Ejemplo: Esto también podría ser de otra forma.Puedes no perdonarlo pero correrte de este sentimiento.

3.6. Sobre sentimientos y acciones del paciente en la sesión o respecto a la relación terapéutica: Afirmaciones respecto a lo que el paciente siente o hace en la sesión o en la relación terapéutica. Ejemplo: Te molesta que pregunte acerca de esto.

3.7. Valoración: El terapeuta realiza un juicio valorativo.

Ejemplo: Esto que te está pasando es muy positivo.

4. (APCA) Afirmaciones para Promover Cursos de Acción: Son recomendaciones y consejos respecto de un curso de acción.

Ejemplo: Sería mejor que lo hablaras con él antes de tomar la decisión.

5. (AT) Afirmaciones sobre el terapeuta: Son autorrevelaciones sobre aspectos de la vida del terapeuta. Pueden ser hechos emocionalmente no significativos, sobre el conocimiento de un tema, hechos de su propia historia, sentimientos, pensamientos y acciones del terapeuta en la sesión.

Ejemplo: Yo también sentiría miedo en esa situación / Me especializo en este tipo de casos / Una vez tuve esa sensación en un barco en medio de una tormenta y estaba realmente preocupado.

6. (AP) Afirmaciones sobre lo que sucede en el proceso terapéutico: Son afirmaciones sobre hechos pasados, 
presentes y futuros del tratamiento cuyo fin es relevar el estado, orientar y estructurar los distintos aspectos del proceso terapéutico.

Ejemplo: En los últimos dos meses pudiste sostener las tareas intersesión.

7. (MI) Meta intervenciones: Afirmaciones referidas a las propias intervenciones del terapeuta. En caso de superposición con AE, se clasifica AP.

Ejemplo: Te quiero decir tres cosas.

8. (FE) Fases y Etapas del tratamiento: Sobre momentos, fases y etapas que sucedieron o que sucederán en el proceso terapéutico. La recapitulación de eventos acontecidos en el proceso terapéutico se puntúa AR. Ejemplo: En un primer momento trabajamos sobre tu ansiedad.

Incluye dos subcategorías:

8.1 Medios y Objetivos del tratamiento: Respecto a los objetivos del tratamiento y sobre las actividades y tareas que deben ser realizadas para lograr la consecución de los objetivos del tratamiento. Ejemplo: Creo que es importante que mantengamos el foco sobre los problemas en tu relación. I Va a ser muy útil que traigas tus anotaciones.

- Si el terapeuta realiza una recapitulación o reformulación sobre creencias, pensamientos, sentimientos y deseos del paciente respecto de los objetivos y medios del tratamiento se puntúa como AR.

- Las Afirmaciones en términos genéricos sobre la conveniencia de establecer ciertos objetivos o medios en función del marco teórico del terapeuta o de la evidencia científica se puntúan como AI.

- Las Hipótesis Explicativas sobre creencias, pensamientos, sentimientos y deseos del paciente sobre objetivos y medios se puntúan como AMA.

8.2.(AD) Afirmaciones respecto al dispositivo que se aplica o aplicará en el tratamiento (individual, vincular, familiar, grupal, combinado, etc.), y afirmaciones respecto a las características, composición, normas, etc. de la institución en la que el tratamiento tiene lugar.

Ejemplo: Una parte va a ser lo que trabajemos acá, (AP) la otra parte será la medicación (AP).

9. (AE) Afirmaciones respecto del encuadre: Son afirmaciones referidas al encuadre del tratamiento. Implican el acuerdo de días, horarios, fechas, así como preguntas sobre lo que sucedió en el momento previo a la sesión o bien sobre datos de la vida social. Ejemplo: El próximo lunes nos veremos a las $18 \mathrm{hs}$. En caso de superposición con AP a, se clasifica AE.

10. (ADC) Afirmaciones de Confirmación: Son afirmaciones que validan o valoran positivamente los pensamientos o emociones del paciente.

Ejemplo: Qué bueno lo que me cuentas.

11. (ASC) Actos sin clasificar:

Incluye seis subcategorías:

11.1. Cuando no se llega a un acuerdo entre tres evaluadores independientes respecto de una UA.

11.2. Saludos de bienvenida y despedida.

11.3. En caso que no quede claro el sentido de lo que dijo el terapeuta.

11.4. Preguntas porque el terapeuta no oyó claramente lo que dijo el paciente. Ejemplo: ¿Qué dijiste?

11.5. Indicaciones al paciente para que repita algo que dijo en caso de que el terapeuta no haya entendido. Ejemplo: Repítelo. 